\title{
Standards im Wettbewerb
}

\author{
Normen und Standards können zur Senkung der Transaktionskosten von markt- \\ lichen Austauschprozessen, vor allem der Senkung des Transaktionsrisikos und \\ der Erhöhung der Vertrauenswürdigkeit, einen wesentlichen Beitrag leisten. \\ Was bedeutet dies für die Weiterentwicklung von EMAS?
}

E:

Von Ulrich Petschow nagementsysteme, die miteinander im Wettbewerb stehen: ISO 14000 als nicht hoheitlicher Standard, und die EG-Öko-Audit-Verordnung (EMAS) als freiwilliger hoheitlicher Standard. Die wesentlichen Unterschiede zwischen diesen beiden Systemen bestehen vor allem im Standortbezug von EMAS (bei ISO Organisationen), in der Frage der kontinuierlichen Verbesserung, der Umweltleistung und dem Eintrag in ein Standortregister (jeweils bei ISO nicht vorgesehen). Auch die Informationsbereitstellung durch eine zu publizierende Umwelterklärung ist allein bei EMAS vorgesehen.

Die Normung von Umweltmanagementsystemen hat generell den Vorteil, daß die Transparenz erhöht und damit Suchkosten verringert werden. Dies hat eine positive Wirkung auf den marktwirtschaftlichen Prozeß, in vielen Bereichen sind Standards geradezu Voraussetzung für das Funktionieren von Märkten. Beispielswiese ist die Erfiillung der ISO 9000ff Standards Für Qualitätsmanagement mittlerweile vielfach eine Vorbedingung für den Zugang zu Märkten (1). Das Problem liegt aber in der Bestimmung der, ,optimalen Menge und Tiefe" an Normen: Durch die Standardisierung kann die Flexibilität soweit eingeschränkt werden, daß die Kosten höher sind als die Nutzen (2).

Die freiwillige Befolgung von Umweltstandards hängt vor allem vom Nutzen ab, der für Unternehmen daraus resultiert. Grundsätzlich werden diesbezüglich die Senkung von Transaktionkosten und die Nutzung von Netzwerkexternalitäten genannt (3). Im Bereich der Umweltqualitätsstandards werden vor allem die Informationsasymmetrien und Unsicherheiten zwischen Verkäufer und Käufer im Hinblick auf die Umweltperformance von Unternehmen, Produkten und Prozessen als bedeutsam angesehen, da diese von Externen schwer zu bewerten ist. Die Anreize zur Nutzung der Standards sind damit vor allem durch den Mechanismus der Reputation (Vertrauen) und die Bereitschaft der Nachfrager, sie zu honorieren, gegeben.

Zentrale (extern orientierte) Vorteile von EMAS sind (4):

- Information der Öffentlichkeit, differenziert nach Fachöffentlichkeit und Konkurrenz,

- rasche Diffusion von Umweltmanagementsystemen, Produkten und Umweltschutzverfahren.

Im Gegensatz zu nicht-publizitätspflichtigen Audits, die vielfach einem Mangel an Glaubwürdigkeit unterliegen, können publizitätspflichtige Audits wie EMAS zu einem vertrauensbildenden Prozeß beitragen.

Von den zur Verfiugung gestellten Informationen und ihrer Qualität hängt die Vertrauenswürdigkeit und letztlich auch der Nutzen für die Unternehmen ab.

\section{Wettbewerbsprozesse auslösen}

Durch die Normung und Standardisierung wird Transparenz hergestellt und die Vergleichbarkeit erhöht. Wenn die Standardisierung nur Mindestvorschriften vorgibt, aber eine öffnung zu anspruchsvolleren Konzepten ermöglicht, können damit zumindest zwei Wettbewerbsprozesse ausgelöst werden:

- Das Wissen um ,best practice“ im Umweltbereich wird verbreitet, da Unternehmen Lösungsansätze von Wettbewerbern identifizieren und gof. übernehmen können. Der Diffusionsprozeß von Innovationen im Umweltbereich wird so beschleunigt. Folge könnte die Unterstiitzung eines ,race to the top" sein (5).

- Externe Anspruchsgruppen erhalten Informationen über die Unternehmen und deren Umweltpraktiken an den jeweiligen Standorten, was zur Vertrauensbildung beitragen kann. Damit wird grundsätzlich auch ein „,benchmarking“" möglich, das der Öffentlichkeit eine Einschätzung über den Umweltschutz an den jeweiligen Standorten ermöglicht.
Ein zentraler Aspekt dieses Wettbewerbs von Umweltmanagementsystemen sind die Informationen, welche Externen, vor allem der interessierten Öffentlichkeit, zur Verfuigung gestellt werden. Um den gesellschaftlich geäußerten Anforderungen gegenüber Standorten und Unternehmen (Stakeholder Ansatz) entsprechen $\mathrm{zu}$ können, ist die (vertrauenswürdige) Publizität ein entscheidender Aspekt, der zu einer kontinuierlichen Entwicklung des Umweltschutzes und damit zur gesellschaftlichen Selbststeuerung beitragen kann. Solche Prozesse sind mit dem ISO Standard nicht möglich, allein die Umwelterklärungen gemäß EMAS können zu diesen, extern angestoßenen, Prozessen beitragen.

Im Wettbewerb zwischen beiden Standards bietet sich insofern eine Arbeitsteilung an: die Mindeststandards werden von der ISO Norm gesetzt, die internationale Gültigkeit hat und internationalen Konventionen entspricht. Das EG-ÖkoAudit-System als zunächst nur europäische (Vorreiter-) Norm könnte Maßstäbe liefern für Ansprüche an die umweltorientierte gesellschaftliche Kooperationsfähigkeit von Unternehmen und damit zu einer Art „Premium ISO“ entwickelt werden. Für ein "race to the top“ müßten die Anreize so gesetzt werden, daß EMAS sich zu dem „Standard“ entwickelt und im besten Fall selbst weiterentwickelt wird. Gelingt dies nicht, besteht die Gefahr eines ,dauerhaft unterstützensbedürftigen Standards" mit erheblichen Verlusten für die Beteiligten (6).

\section{Konsequenzen für EMAS}

Entscheidend ist somit, daß für die Unternehmen die erweiterten Maßstäbe von EMAS mit Nutzen verbunden sind. Anreize dazu müssen wesentlich durch Anspruchsgruppen formuliert werden.

Da nur EMAS aufgrund der Publizitätspflicht überhaupt in der Lage ist, Informationsasymmetrien zu verändern und so die positiven Wirkungen von Standards zur Geltung zu bringen, muß dieser Aspekt in den Vordergrund gestellt werden. Dies bedeutet aber auch, daß die vermittelten Informationen transparent, vertrauenswürdig und vermittelbar sein müssen.

Dabei sind vor allem folgende Aspekte von Bedeutung:

- Der Abnehmer von Endprodukten muß in der Lage sein, Schlußfolgerungen aus der EMASTeilnahme ziehen zu können. Dies könnte durch eine Integration z.B. in Produkt-Umweltzeichen erfolgen (vgl. Rubik in diesem Heft). 
Abnehmer aus dem Unternehmensbereich müssen unter Nutzung von Netzwerkvorteile in der Lage sein, weitere vermittelbare Informationen aus der EMAS-Teilnahme zu erhalten.

- Die Öffentliche Hand als Nachfrager könnte ggf. die Kombination von EMAS und Produktlabel zum Standard für ihr Einkaufsverhalten machen und damit einen ,,pull“-Effekt auslösen.

- Die Aufsichtsbehörden können im Gegenzug zum Aufbau eines leistungsfähigen überprüfbaren Öko-Audit-Systems Deregulierungspotentiale nutzen.

- Von Seiten der EU müssen die entsprechenden Anreize gesetzt werden, die diesen Standard im Wettbewerb mit z.B. der ISO Norm stützen. EMAS als „Premium-ISO“ müßte zugleich derart transparent sein, daß auch die Unterschiede vermittelbar sind und sich somit Vorreiterunternehmen durch die Ausgestaltung von EMAS absetzen können. Dies könnte durch entsprechende Benchmarking-Aktivitäten wesentlich unterstïtzt werden.

\section{Anmerkungen}

(1) Kassebohm, K. et al.: Auditierung und Zertifizierung im Brennpunkt wirtschaftlicher und rechtlicher Interessen. In: ZfB (1994), S. $692-715$.

(2) Eger, T.: Wieviel Normung braucht der Markt? Ökonomische Aspekte der Vertragsfreiheit. In: Jahrbuch Ökonomie und Gesellschaft Nr. 11 (1995): Markt, Norm und Moral.

(3) Leveque, F.: Standards and standard-setting processes in the field of environment. In: R. Hawkins et al. (1995): Standards, Innovation and Competitiveness. The Politics and Economics of Standards in Natural and Technical Environments.

(4) Vgl. z.B. Sachverstündigenrat für Umwelffragen: Umweltgutachten 1998, Bundestags-Drucksache 13/10195, S.151ff.

(5) Clousen, J., U. Petschow, J. Behnsen: Umwelterklärungen als Innovationsbarometer. IÖW Schriftenreihe Nr. 114/97.

(6) Helbig, J. et al.: Wirtschaftspolitische Aspekte einer wachsenden Bedeutung von Normen im (betrieblichen) Umweltschutz. Tübingen 1997

\section{Der Autor}

Ulrich Peischow ist wissenschaftlicher Mitarbeiter am IÖW im Forschungsfeld Unweltökonomie und Umweltpolitik.

Kontakt: IÖW, Giesebrechistr. 13, 10629 Berlin. Tel. 030/88 45 94-0, Fax 030/882 54 39, E-mail: mailbox@ioew.b.eunet.de

Die Entwicklung und Implementation von EMAS auf EU-Ebene

\section{Politikinnovation mit Hindernissen}

\section{Mit der EMAS-Verordnung wurde die europäische Umweltpolitik um ein Kon- zept bereichert, das einige grundlegende Innovationen beinhaltet. Wie konnte sich EMAS im Prozeß der europäischen Politikformulierung durchsetzen? Wie effektiv ist die Implementation der Verordnung auf nationaler Ebene bisher?}

$D_{\text {sim }}$ Von Christoph Knill e Entwicklung von EMAS erfolgte im Kontext einer generellen strategischen Umorientierung in der europäischen Umweltpolitik, die sich seit Ende der achtziger Jahre beobachten läßt und speziell im fünften Umweltaktionsprogramm von 1993 nochmals bekräftigt wird. Im Kern dieser Trendwende steht die zunehmende Ersetzung und Ergänzung traditioneller hierarchischer Steuerungsinstrumente durch neue Regulierungsansätze, welche auf prozedurale Regulierung, Selbststeuerung, freiwillige Vereinbarungen und die Beteiligung der Öffentlichkeit abzielen (1). Wichtige Maßnahmen, die im Rahmen dieser neuen ,bottom-up“ Steuerung verabschiedet wurden, sind neben der EMAS-Verordnung die Richtlinien über den freien Zugang zu Umweltinformationen, die EcoLabel-Verordnung und die Rahmenrichtlinie zur Luftqualität.

Die Gründe für diese strategische Trendwende liegen hauptsächlich in den Implementationsdefiziten, welche sich im Rahmen der hierarchischen ,top-down“ Steuerung in verstärkter und andauernder Form gezeigt hatten. Diesem Problem soll mit der Verwendung von ,bottom-up“ Konzepten in zweifacher Hinsicht abgeholfen werden. Einerseits sind die neuen Instrumente von ihrem Design her institutionell weniger ,einschneidend", d.h. ihre Vorgaben sind "offener" und lassen sich somit leichter in bestehende regulative Arrangements auf nationaler Ebene integrieren. Administrative Widerstände bei der Implementation europäischer Vorgaben sind somit weniger wahrscheinlich. Zum zweiten sind die „bottom-up“ Ansätze speziell darauf ausgerichtet, die breite Unterstuitzung nationaler Akteure zu mobilisieren, d.h. es wird stärker auf die Interessenlage der Politikadressaten Bezug genommen. Je höher die Unterstiutzung ,von unten", so das Kalkuil, desto stärker ist wiederum der Druck auf nationale Implementeure, europäische Vorgaben effektiv umzusetzen. Die- se Annahmen werden im wesentlichen durch frühere Ergebnisse der Implementationsforschung bestätigt (2).

Allerdings sind auch ,bottom-up“ Instrumente trotz der institutionell weniger weitreichenden Vorgaben nicht frei von administrativen Implikationen auf nationaler Ebene und somit von Widerständen im Prozeß der Politikformulierung und Implementation.

\section{Umstriftene Einführung}

Die Entwicklung eines europäischen EMAS-Konzeptes basiert auf einem Vorschlag der Kommission, welcher wiederum von einer entsprechenden Initiative der Internationalen Handelskammer inspiriert war. Bereits während erster Diskussionen auf Kommissionsebene hatte sich gezeigt, daß die Mitgliedstaaten nicht bereit waren, die Einfuihrung von EMAS als rechtlich verbindliches Politikinstrument zu akzeptieren. Aufgrund dieser einhelligen Kritik seitens der Mitgliedstaaten entschloß sich die Kommission, EMAS als freiwilliges Instrument zu konzipieren. Als Folge dieses bereits im Vorfeld stark veränderten und nationalen Interessen entgegenkommenden Konzeptes fand der Vorschlag der Kommission breite Zustimmung im Ministerrat. Lediglich die Bundesrepublik sperrte sich zunächst vehement gegen die Maßnahme, während sich Großbritannien sehr schnell als stärkster Verfechter der Verordnung entpuppte. Wie bei vielen anderen Entscheidungen zur europäischen Umweltpolitik war damit auch die Entwicklung von EMAS durch das Aufeinanderprallen zweier gegensätzlicher nationaler Regulierungsvorstellungen geprägt.

Die wesentlichen Elemente der Verordnung entsprachen weitgehend dem flexiblen, auf prozeduralen Regeln und industrieller Selbstregulierung basierenden britischen Ansatz. Daher waren die mit der Maßnahme verbundenen institutionellen Anpassungskosten im Falle Großbritanniens sehr gering. Überdies hatten die Briten kurz zuvor einen eigenen nationalen Standard für Umwelt- 
(c) 20I0 Authors; licensee IÖW and oekom verlag. This is an article distributed under the terms of the Creative Commons Attribution Non-Commercial No Derivates License (http://creativecommons.org/licenses/by-nc-nd/3.o/), which permits unrestricted use, distribution, and reproduction in any medium, provided the original work is properly cited. 